\title{
Eye-Specific Projections of Retinogeniculate Axons Are Altered in Albino Mice
}

\author{
Alexandra Rebsam, ${ }^{1}$ Punita Bhansali, ${ }^{1}$ and Carol A. Mason ${ }^{1,2,3}$ \\ ${ }^{1}$ Department of Pathology and Cell Biology, ${ }^{2}$ Department of Neuroscience, and ${ }^{3}$ Department of Ophthalmology, College of Physicians and Surgeons, \\ Columbia University, New York, New York 10032
}

The divergence of retinal ganglion cell (RGC) axons into ipsilateral and contralateral projections at the optic chiasm and the subsequent segregation of retinal inputs into eye-specific domains in their target, the dorsal lateral geniculate nucleus (dLGN), are crucial for binocular vision. In albinism, affected individuals exhibit a lack or reduction of pigmentation in the eye and skin, a concomitant reduced ipsilateral projection, and diverse visual defects. Here we investigate how such altered decussation affects eye-specific retinogeniculate targeting in albino mice using the C57BL/6 $\mathrm{Tyr}^{c-2 J / c-2 J}$ strain, in which tyrosinase, necessary for melanogenesis, is mutated. In albino mice, fewer RGCs from the ventrotemporal (VT) retina project ipsilaterally, reflected in a decrease in cells expressing ipsilateral markers. In addition, a population of RGCs from the VT retina projects contralaterally and, within the dLGN, their axons cluster into a patch separated from the contralateral termination area. Furthermore, eye-specific segregation is not complete in the albino dLGN and, upon perturbing postnatal retinal activity with epibatidine, the ipsilateral projection fragments and the aberrant contralateral patch disappears. These results suggest that the defects in afferent targeting and activity-dependent refinement in the albino dLGN arise from RGC misspecification together with potential perturbations of early activity patterns in the albino retina.

\section{Introduction}

The partial decussation of retinal ganglion cell (RGC) axons at the optic chiasm midline ensures that targets on each side of the brain receive inputs from both eyes. In their primary target, the dorsal lateral geniculate nucleus (dLGN), RGC afferents from each eye segregate into distinct territories. This precise organization of eye-specific inputs is crucial for binocular vision, and during development is dependent on both molecular factors and neural activity (Huberman et al., 2008).

Molecular determinants of chiasmatic decussation have been identified (Petros et al., 2008). The transcription factor Zic2 and the guidance receptor EphB1 expressed in RGCs from ventrotemporal (VT) retina direct the ipsilateral projection (Herrera et al., 2003; Williams et al., 2003). Contralateral markers such as the transcription factor Islet2 (Pak et al., 2004) and the adhesion molecule NrCAM (Williams et al., 2006) are expressed in a pattern complementary to Zic2 and EphB1 and are important for late-born VT RGCs to cross the midline.

Received Oct. 5, 2011; revised Nov. 22, 2011; accepted Feb. 19, 2012.

Author contributions: A.R. and C.A.M. designed research; A.R. and P.B. performed research; A.R. analyzed data; A.R., P.B., and C.A.M. wrote the paper.

This work was supported by NIH Grants EY012736 and EY015290, the Gatsby Initiative in Brain Circuitry (C.A.M.), and the Fondation pour la Recherche Medicale and the Human Frontier Science Program (A.R.). We thank Takeshi Sakurai, Ray Guillery, Patricia Gaspar, Wes Grueber, and members of the Mason Lab for helpful discussions and critical review of the manuscript.

Correspondence should be addressed to either Carol A. Mason, Department of Pathology and Cell Biology, College of Physicians and Surgeons, Columbia University, 630 W 168th Street, New York, NY 10032. E-mail: cam4@columbia.edu or alexandra.rebsam@inserm.fr.

A. Rebsam's present address: INSERM UMR-S839, Institut du Fer à Moulin, Paris, France.

DOI:10.1523/JNEUROSCI.5050-11.2012

Copyright $\odot 2012$ the authors $\quad 0270-6474 / 12 / 324821-06 \$ 15.00 / 0$
Previously, we addressed how impaired decussation affects retinogeniculate targeting: in EphB1 $1^{-/-}$mice, fewer RGCs project ipsilaterally but innervate the appropriate targeting zone (Rebsam et al., 2009). We next turned to analyzing eye-specific targeting in the albino mouse, another model with altered decussation. Albinism is a hypopigmentation disorder due to lack of melanin in the eye only (ocular albinism), or in the eyes, skin, and hair (oculocutaneous albinism) (Oetting, 1999). A universal phenotype of albinism is a decreased ipsilateral projection resulting in perturbed binocular vision (Creel et al., 1990; Apkarian, 1996). We used $T y r^{c-2 J / c-2 J}$ mice, in which tyrosinase, crucial for melanin synthesis, is mutated (Le Fur et al., 1996). All albino mouse strains have a reduced ipsilateral projection to the dLGN (Guillery et al., 1973; LaVail et al., 1978; Dräger and Olsen, 1980; Rice et al., 1995), but no other dLGN targeting defects have been described (Guillery et al., 1973; Cronin et al., 2003). Moreover, in $\mathrm{Tyr}^{c-2 J / c-2 J}$ mice, fewer RGCs express Zic2 in the VT retina (Herrera et al., 2003), mirroring the reduced ipsilateral projection.

Here, we show that the reduced ipsilateral projection in albino mice is not characterized by a simple misrouting of retinal axons to the contralateral dLGN with retention of their proper targeting pattern, as in EphB1 $1^{-/-}$mice (Rebsam et al., 2009). Instead, some VT RGCs from the contralateral eye terminate near the target zone of VT RGCs that normally project contralaterally in pigmented mice, but as a cluster separated from the principle contralateral projection. The reduction of ipsilateral fibers in the albino correlates with fewer RGCs expressing Zic2 and EphB1, but not with an increase in RGCs expressing contralateral markers. Moreover, as in EphB1 mutants, eye-specific segregation in albino mice is impaired under normal conditions, and the formation of the aberrant patch of RGC axons from VT retina is 

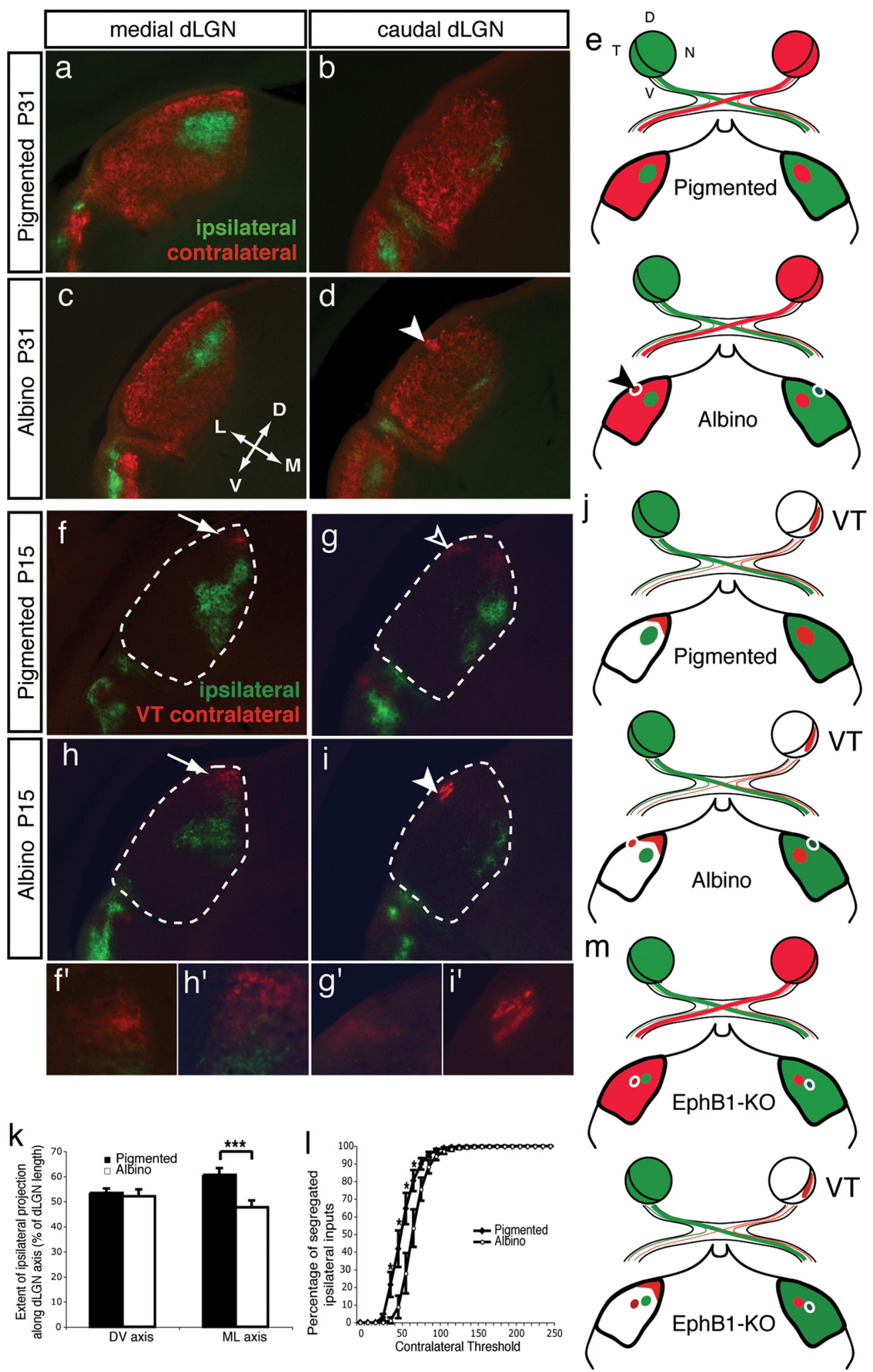

Figure 1. Retinogeniculate projections in pigmented and albino mice. $\boldsymbol{a}-\boldsymbol{j}$, Coronal sections through the dLGN after whole-eye anterograde tracing at P31 ( $\boldsymbol{a}-\boldsymbol{e}$ ) or VT retina anterograde tracing in one eye and whole-eye tracing in the other eye at $P 15(\boldsymbol{f}-\boldsymbol{j}) \cdot \boldsymbol{a}, \boldsymbol{b}$, In pigmented mice, the ipsilateral projection (green) forms a patch in the dorsocentral dLGN surrounded by contralateral terminations (red). $\boldsymbol{c}, \boldsymbol{d}$, In albino mice, the ipsilateral termination zone is reduced in size (c), and a segregated patch of fibers from the contralateral eye is positioned in the caudal dLGN adjacent to the optic tract and dorsal tip of the dLGN (d, arrowhead). $\boldsymbol{f}, \boldsymbol{g}$, In pigmented mice at P15, late-born VT RGCs project contralaterally to the dorsal tip of the dLGN in medial sections $(\boldsymbol{f}$, arrow; $\boldsymbol{f})$ and more laterally in caudal sections $(\boldsymbol{g}$, arrowhead; $\boldsymbol{g})$ in pigmented mice. $\boldsymbol{h}, \boldsymbol{i}$, The aberrant patch in albino mice arises from the VT retina (i, arrowhead) and is located adjacent to the normal contralateral projection from the VT retina $\left(\boldsymbol{g}\right.$, arrowhead). $\boldsymbol{f}^{\prime}, \boldsymbol{g}^{\prime}, \boldsymbol{h}^{\prime}, \boldsymbol{i}^{\prime}$, Higher magnification of the VT contralateral projection in pigmented and albino dLGN shown in $\boldsymbol{f}-\boldsymbol{i}$, respectively. $\boldsymbol{e}, \boldsymbol{j}, \boldsymbol{m}$, Diagrams depict RGC termination regions in the dLGN after anterograde tracing in pigmented, albino, and EphB1-KO mice. Left side corresponds to photomicrographs $\boldsymbol{a}$-i and previous result on EphB1-KO mice in Rebsam et al. (2009). Right side represents the opposite side (picture not shown). $\boldsymbol{j}$, The ipsilateral projection, solely arising from VT retina, is entirely labeled by VT injections. Thus, targeting to the dLGN on the opposite side of the one described in $\boldsymbol{f}$ and $\boldsymbol{h}$ is an inverted mirror image of the one described in $\boldsymbol{a}$ and $\boldsymbol{c}$, respectively (data not shown). $\boldsymbol{k}$. Extent of the ipsilateral projection expressed as a percentage of length along the (Figure legend continues.) 
dependent on normal retinal activity. Finally, perturbation of retinal activity with epibatidine in albino animals produces changes nearly opposite to those seen in pigmented animals, suggesting possible abnormalities in early patterns of neural activity in the albino retina.

\section{Materials and Methods}

Animals. Wild-type C57BL/6J mice and albino mice lacking tyrosinase (C57BL/6J Tyr ${ }^{c-2 J / c-2 J}$ from Jackson Laboratory) were maintained in a timed-pregnancy breeding colony at Columbia University. Pigmented and albino mice were generated from crosses between $\mathrm{Tyr}^{+} / \mathrm{Tyr}^{c-2 J}$ and $\mathrm{Tyr}^{c-2 J} / \mathrm{Tyr}^{c-2 J}$ mice. We use the terms pigmented and albino to refer to animals of the genotypes $\mathrm{Tyr}^{+} / \mathrm{Tyr}^{+}$or $\mathrm{Tyr}^{+} / \mathrm{Tyr}^{c-2 J}$ and $\mathrm{Ty} r^{c-2 J} / \mathrm{Tyr}^{c-2 J}$, respectively (LaVail et al., 1978). E0 was defined as midnight before a plug was found and $\mathrm{P} 0$ is the day of birth, and both sexes were studied. All animal procedures followed the regulatory guidelines of the Columbia University Institutional Animal Care and Use Committee.

Anterograde labeling of retinogeniculate projections. P13 and P29 mice were anesthetized intraperitoneally with ketamine-xylazine (100 and 10 $\mathrm{mg} / \mathrm{kg}$, respectively, in $0.9 \%$ saline). For whole-eye injection, the eye was extruded by using two fingers and $2-4 \mu \mathrm{l}$ of $0.2 \%$ cholera-toxin subunit B (CTB) conjugated to AlexaFluor 594, 488 (Invitrogen) diluted in $1 \%$ DMSO was injected into the eye intravitreally with a glass micropipette. To make focal injections, 300-400 $\mathrm{nl}$ of $4 \%$ liquid DiI in $10 \%$ sucrose (the liquid DiI solution consists of $10 \%$ DiI in dimethylformamide) or CTB-AlexaFluor 594 (0.1\% in 0.5\% DMSO, 10\% sucrose solution) was injected beneath the scleral margin into the VT retina through a glass micropipette linked to a Picospritzer II (General Valve Corporation). The ventrotemporal position was determined using the eyelid junction and head position. After $2 \mathrm{~d}$, mice were anesthetized and perfused transcardially with $4 \%$ paraformaldehyde (PFA) in $0.1 \mathrm{~m}$ phosphate buffer before dissection of brain and retinas. Brains were postfixed overnight, embedded in 3\% agarose, and sectioned coronally with a vibratome $(100 \mu \mathrm{m})$. Sections were mounted in Gelmount (Biomeda).

Epibatidine injection. Postnatal mice were anesthetized on ice. The skin was cut open at the eyelid junction to access the eye. Every $24 \mathrm{~h}$ from P3 to $\mathrm{P} 6,0.5-1 \mu \mathrm{l}$ of $1 \mathrm{~mm}$ epibatidine in $0.9 \%$ saline was injected intravitreally into both eyes through the same injection site. Pups were returned to their mother after recovery from anesthesia.

In situ hybridization and immunostaining. Heads from E15.5 and E17.5 embryos were fixed overnight or $2 \mathrm{~h}$ for Islet2 immunostaining in $4 \%$ PFA, cryoprotected in $10 \%$ sucrose in PBS overnight, and subsequently frozen in TissueTek OCT compound using cold isopentane $\left(-55^{\circ} \mathrm{C}\right)$. Coronal sections $(20 \mu \mathrm{m})$ were cut with a cryostat, mounted on Superfrost slides, and processed for in situ hybridization as described by Schaeren-Wiemers and Gerfin-Moser (1993). Probes for EphB1 (Williams et al., 2003) and NrCAM (Williams et al., 2006) were synthesized using Digoxygenin RNA labeling mix (Roche). The sections were then processed for immunohistochemistry. Ten percent normal goat serum (NGS) in $0.2 \%$ Triton was used as blocking solution. A rabbit anti-Zic2 antibody (1/10,000; a gift from Dr. Steve Brown, University of Vermont, Burlington, VT) and a rabbit anti-Islet2 antibody (1/1000; a gift from Dr. Thomas Jessell, Columbia University, New York, NY) were used as primary antibodies, and a goat anti-rabbit Cy3 (1/500; Jackson Immunoresearch) was used as a secondary antibody in $1 \%$ NGS, $0.2 \%$ Triton in PBS. Sections were mounted in Gelmount.

(Figure legend continued.) DV and ML axes of the dLGN. Ipsilateral fibers cover more territory along the ML dLGN axis in albino mice compared with pigmented mice. I, Segregation plot. Percentage of segregated inputs as a function of contralateral threshold (ipsilateral threshold is fixed). In albino mice, ipsilateral fibers are less segregated from contralateral fibers (more overlap) than in pigmented mice. D, Dorsal; $\mathrm{V}$, ventral; $\mathrm{M}$, medial; $\mathrm{L}$, lateral; $\mathrm{N}$, nasal; $\mathrm{T}$, temporal. Axes correspond to the orientation used for quantifications along the DV and ML axes, but are slightly tilted compared with actual brain orientation. Error bars are SEM. ${ }^{*} p<0.05$, $* * * p<0.001$.
Microscopy. Imaging of embryonic retinas and brain sections was done with Axiovision software through Axiophot camera and a Zeiss Axioplan 2 microscope.

Analysis of the number, distribution, and segregation of ipsilateral fibers. Quantification of eye-specific terminations in Figures 1 and 4 were performed using MetaMorph software on $10 \times$ images and statistical analysis using Student's $t$ test were performed as previously described (Rebsam et al., 2009).

\section{Results}

The number, distribution, and segregation of ipsilateral fibers are altered in the albino mouse dLGN

Using whole-eye labeling with fluorescent CTB to trace retinogeniculate axons (Fig. 1e) and computer-assisted quantification, we found that at P31, the ipsilateral RGC projection in albino mice $(n=6)$ targets the dLGN core as in wild-type (pigmented) dLGN (Fig. 1a,c) but is reduced, as expected, by $48 \%$ compared with pigmented mice $(n=11)$ (pigmented, $17.2 \%$, and albino, 9.7\% of dLGN territory is occupied by ipsilateral terminal arbors; $\left.p=9.3 \times 10^{-5}\right)$. The reduced ipsilateral projection in the albino $\mathrm{dLGN}$ is confined to a smaller zone along the ML axis and has a more elongated shape (Fig. 1c) compared with pigmented mice (Fig. 1a). This pattern is supported by quantification of RGC terminations, indicating a reduced extension of the ipsilateral projection along the ML axis but not the DV axis (Fig. $1 k$ ). Moreover, eye-specific segregation in the albino dLGN is incomplete, reflected in a significantly greater overlap between ipsilateral and contralateral terminal arbors at P31 compared with pigmented mice (Fig. 1l).

\section{RGCs from VT retina in albinos project within the contralateral input-recipient zone as a distinct patch}

The most striking feature of the albino retinogeniculate projection is a patch of axon terminations from the contralateral eye that forms within the contralateral RGC-recipient zone but is separated from the normal contralateral projections from non-VT and VT retina (Fig. 1d). The patch of axons is situated in the caudal dLGN, adjacent to the optic tract and ventrolateral to the afferent zone of VT RGCs that are late-born and that project contralaterally in pigmented and albino mice (Fig. $1 b, d$ ). This phenotype is fully penetrant, as we observed this patch in every individual albino brain.

In pigmented mice, DiI injections made at P15 and restricted to the VT retina (Fig. 1j) labeled a projection to the core of the dLGN on the ipsilateral side (data not shown) and the dorsal tip of the dLGN on the contralateral side (Fig. $\left.1 f, f, h, h^{\prime}\right)$, where the late-born contingent of VT RGCs terminates (Dräger, 1985; Pfeiffenberger et al., 2006). In albino mice, such VT injections revealed that the aberrant patch is composed of fibers from the contralateral VT retina (Fig. $\left.1 i, i^{\prime}, g, g^{\prime}\right)$. This pattern of projection suggests that in the albino, VT RGC axons project to a site near the topographically correct terminal zone in the dLGN of contralateral VT RGC axons. However, because these RGCs form a cluster of terminals separated from the other contralateral projections, these RGCs may be differentially specified, expressing a combination of markers different from those in the normal contralaterally projecting VT and non-VT RGCs (Fig. $1 j$ ).

\section{Changes in ipsilateral but not contralateral marker expression in the albino VT retina}

We then assessed whether the expression pattern of markers of RGC specification and guidance fate are altered in the albino, 
especially in VT retina (Fig. 2). As previously described, the number of VT RGCs expressing the ipsilateral determinant $\mathrm{Zic} 2$ is reduced in albino mice (Fig. 2a2,b2) (Herrera et al., 2003). We found that the number of RGCs expressing the EphB1 receptor (Fig. 2a1,b1) is also reduced in the albino retina at E15.5, mirroring the reduction of Zic2-expressing cells (Fig. 2a,b). For contralateral markers, however, NrCAM expression at E15.5 (Fig. $2 c, d$ ) and E17.5 (Fig. 2e,f) and Islet2 expression at E17.5 (Fig. $2 g, h$ ) are similar in pigmented and albino mice. This result suggests that the reduction in the number of RGCs with an ipsilateral identity is not accompanied by an increase in RGCs expressing known contralateral markers.

Perturbation of retinal waves has an unusual effect on RGC fiber refinement in albino mice

Spontaneous waves of activity in the retina are important for eye-specific segregation (Huberman et al., 2008; Xu et al., 2011). As retinal waves rely on cholinergic transmission through the first postnatal week, ocular injections of the nicotinic acetylcholine receptor agonist epibatidine perturbs retinal waves through receptor desensitization (Feller et al., 1996), increasing firing in some RGCs and silencing others (Cang et al., 2005; Sun et al., 2008). We have previously shown that disrupting retinal activity during the first postnatal week has lasting effects on the distribution and segregation of ipsilateral terminals (Rebsam et al., 2009). After epibatidine treatment in wild-type mice (Fig. $3 b)$, ipsilateral fibers are distributed over a larger territory along the DV and ML axis compared with saline-treated wild-type mice (Figs. $3 a, b ; 4 b$ ) and are less segregated from contralateral fibers (Figs. $3 a^{\prime}, b^{\prime} ; 4 c, d$ ). However, the proportion of dLGN occupied by ipsilateral fibers is not significantly increased (Fig. $4 a$ ).

In albino mice treated with epibatidine $(n=7)$, ipsilateral projections fragment into distinct patches (often three) along the DV axis of the dLGN (Fig. 3d) and are distributed along a wider extent of the DV axis compared with saline-treated albino mice $(n=5$; Figs. $3 c, d ; 4 b)$. No significant differences were observed between saline- and epibatidine-treated albino mice in the percentage of the dLGN area occupied by ipsilateral fibers, in extent along the ML axis, nor in eye-specific segregation (Figs. $3 c^{\prime}, d^{\prime} ; 4$ ), even though segregation is incomplete in the albino under normal spontaneous activity compared with pigmented mice (Fig. 3a', ') ). Importantly, binocular epibatidine treatment in albino mice prevents the formation of the aberrant patch formed by contralateral fibers (Fig. 3e,f). Thus, epibatidine treatment has an almost opposite effect on distribution and segregation of RGC afferents in albino mice compared with wild-type mice. Furthermore, these data indicate that retinal activity is required for the clustering of the aberrant contralateral fibers arising from VT RGCs in albino mice.

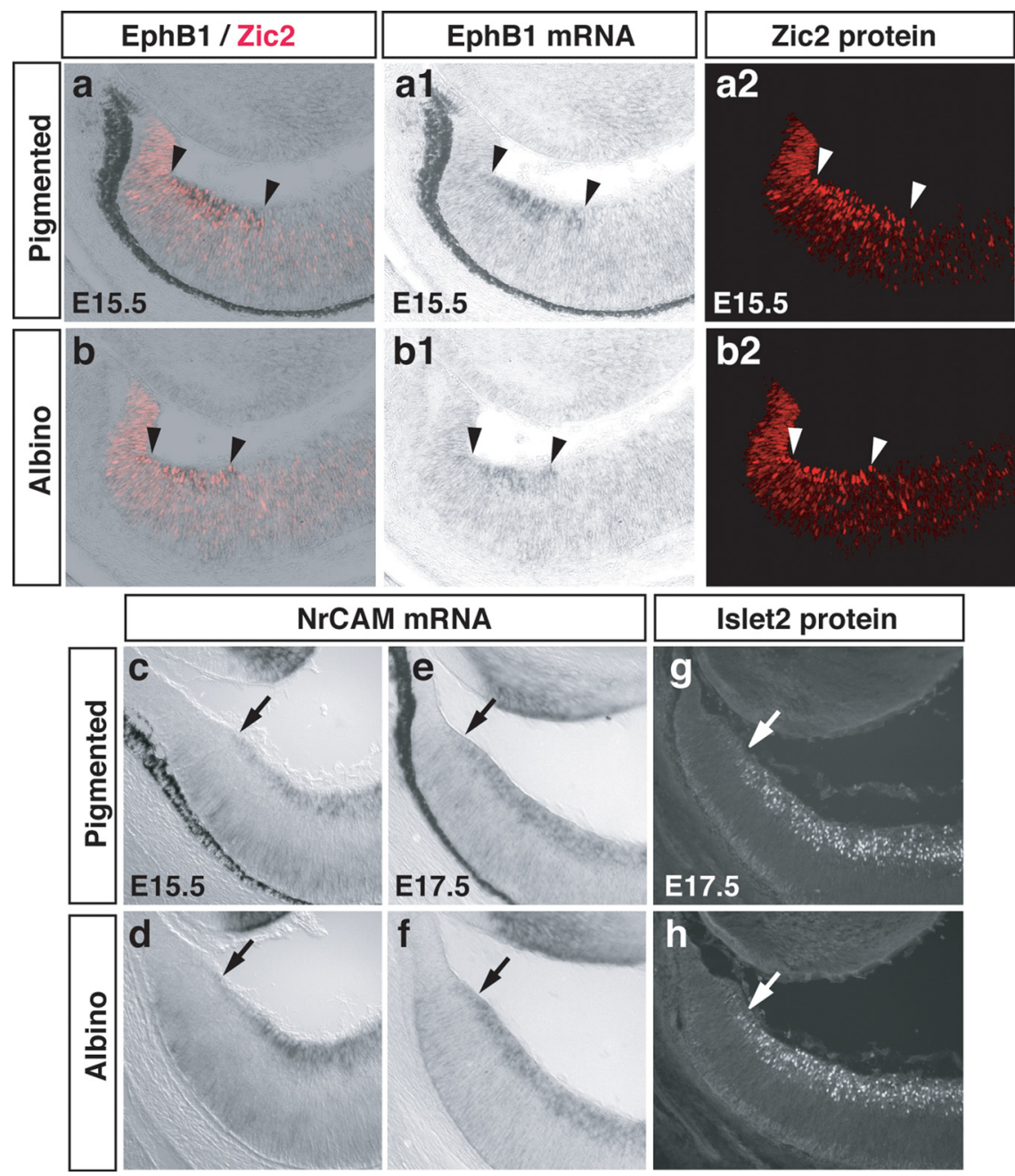

Figure 2. $\quad \boldsymbol{a}, \boldsymbol{b}, \mathrm{EphB1}, \mathrm{Zic} 2$, NrCAM and Islet2 expressions in VT retina. EphB1 (in situ hybridization, black) is coexpressed with expressed in fewer RGCs of the VT retina compared with pigmented mice $(\boldsymbol{a})$ (arrowheads). Separated images for EphB1 $(\boldsymbol{a} \mathbf{1}, \boldsymbol{b} \mathbf{1})$ and Zic2 $(\boldsymbol{a} \mathbf{2}, \boldsymbol{b 2})$. NrCAM and Islet2 expression in the VT retina is similar in pigmented and albino mice at E15.5 ( $\boldsymbol{c}, \boldsymbol{d}$ for NrCAM) and E17.5 (e, $\boldsymbol{f}$ for NrCAM and $\boldsymbol{g}, \boldsymbol{h}$ for Islet2).

\section{Discussion}

We have examined how altered RGC decussation at the optic chiasm in albino mice affects targeting in the dLGN. In albino mice, fewer VT RGC axons project to the ipsilateral side and some contralaterally projecting VT RGCs form an aberrant patch that is separated from the normal contralateral and ipsilateral cohorts in an activity-dependent manner. In addition, in albino mice, retinal activity disruption by epibatidine induces the fragmentation of the ipsilateral projection into patches without further perturbing eye-specific segregation.

Albino and EphB1 ${ }^{-/-}$mice have a reduced ipsilateral projection (Dräger and Olsen, 1980; Williams et al., 2003). In both mutants, a small cohort of VT RGCs projects contralaterally and forms a patch of terminations separated from the surrounding projections. However, whereas the aberrant patch in EphB1 $1^{-/-}$ mice is located adjacent to the normal ipsilateral projection, the aberrant patch in the albino terminates in a contralateralrecipient territory, adjacent to the zone where late-born contralateral RGCs from VT retina project. These results suggest that abnormal RGC specification may be responsible for the aberrant retinogeniculate projection pattern in the albino. Indeed, the al- 


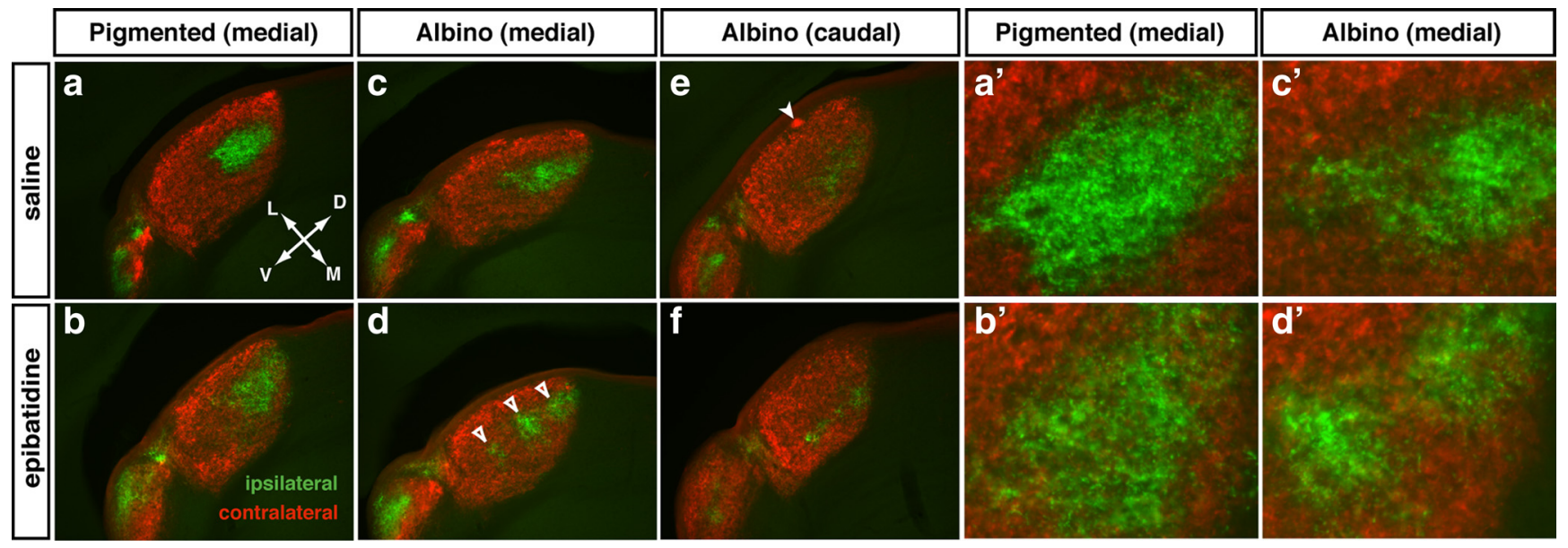

Figure 3. Retinogeniculate projections at P31 after binocular epibatidine treatment from P3 to P6. $\boldsymbol{a}, \boldsymbol{b}$, In pigmented dLGN after epibatidine treatment (b), ipsilateral RGC fibers are more scattered, extend over a greater area, and overlap more with contralateral fibers $\left(\boldsymbol{a}^{\prime}, \boldsymbol{b}^{\prime}\right)$ compared with saline-treated mice $(\boldsymbol{a}) . \boldsymbol{c}-\boldsymbol{f}$, In contrast, in the albino dLGN, epibatidine treatment induces fragmentation of the ipsilateral inputs into distinct patches (d, arrowheads), with RGC fibers extending further along the DV axis of the dLGN. In addition, aggregation of the abnormal contralateral patch arising from VT retina and positioned near the optic tract is abolished after epibatidine treatment in albino mice (e, arrowhead, compare with $\boldsymbol{f})$. $\boldsymbol{a}^{\prime}-\boldsymbol{d}^{\prime}$, Higher magnification of ipsilateral fibers in the dLGN as seen in $\boldsymbol{a}-\boldsymbol{d}$, respectively. D, Dorsal; V, ventral; M, medial; L, lateral. Axes correspond to the orientation used for quantifications along DV and ML axes, but are slightly tilted compared with actual brain orientation.
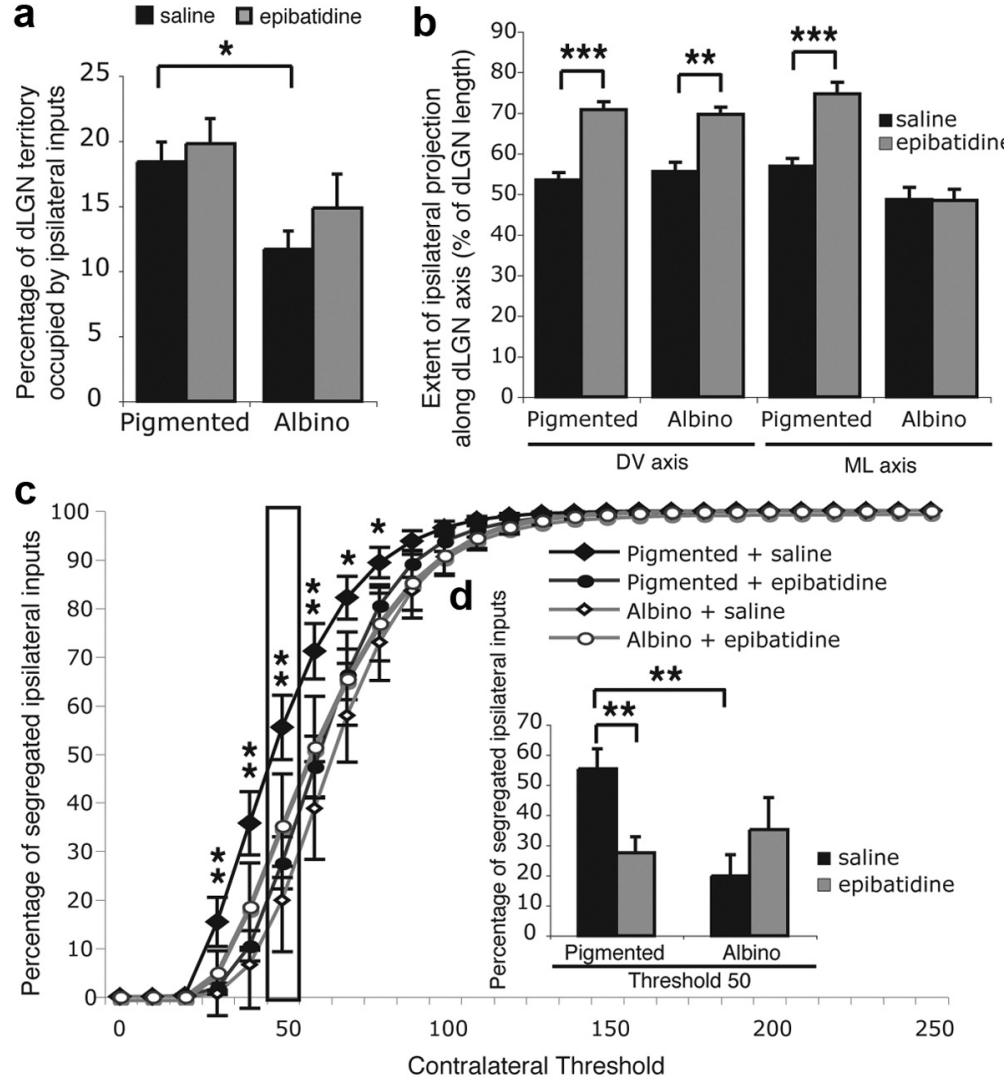

Figure 4. Comparison of ipsilateral projections in pigmented and albino mice after epibatidine treatment. $\boldsymbol{a}$, In salinetreated mice, the area of the dLGN occupied by ipsilateral fibers is reduced in albino compared with pigmented mice. In both cases, binocular epibatidine treatment had no significant effect on the percentage of dLGN occupied by ipsilateral fibers. $\boldsymbol{b}$, The ipsilateral projection extends along the DV axis in both pigmented and albino mice after epibatidine treatment, but only epibatidine-treated pigmented mice show a significant extension of the ipsilateral projection along the ML axis of the dLGN. c, Segregation plot. Percentage of segregated inputs as a function of the contralateral threshold (ipsilateral threshold is fixed). $\boldsymbol{d}$, Columnar representation of the values in $\boldsymbol{c}$ for the contralateral threshold set at 50 . Segregation of ipsilateral inputs in saline-treated albino mice is significantly impaired compared with pigmented mice. Segregation of ipsilateral inputs is further impaired in the dLGN of pigmented mice after epibatidine treatment. For albino mice treated with epibatidine, an opposite trend is observed, toward an increase in the segregation of ipsilateral fibers, but is not significant. Error bars are SEM. ${ }^{*} p<0.05,{ }^{* *} p<0.01,{ }^{* * *} p<0.001$. bino retina contains fewer Zic2 (Herrera et al., 2003) and EphB1-positive RGCs, consistent with the regulation of EphB1 by Zic2 (García-Frigola et al., 2008; Lee et al., 2008). Because our previous results showed that albino VT RGCs display crossed RGC behaviors in vitro (Marcus et al., 1996), we expected to see an increase in crossed markers, but did not. Further, we could not determine whether the VT RGCS that form the patch are RGCs that are normally specified to project ipsilaterally and have changed fate or whether they are RGCs that were already fated to project contralaterally. In both cases, these RGCs might lack or aberrantly express a combination of transcription factors and guidance molecules differently from that in wild-type contralateral VT RGCs and thus project and terminate as a separate patch in the albino. The expression of transcription factors or surface molecules could in turn be regulated by activity (Itoh et al., 1995; Hanson and Landmesser, 2004), explaining why the patch disappears after blockade of correlated retinal activity.

VT RGCs in albino retina may have such an altered transcriptional profile due to defects in the pace of proliferation (Ilia and Jeffery, 1996; Rachel et al., 2002), supported by the finding that tyrosinase is required only during the generation of ipsilateral RGCs for the development of a normal projection (Cronin et al., 2003). If the aberrant population of VT RGCs are born too early or too late, they would be specified to express molecular determinants that would render the RGCs unrec- 
ognizable to the other VT axons and would consequently form their own termination zone. Understanding differences in the molecular code of the different populations of VT RGCs will be necessary to further dissect the molecular mechanisms of the targeting defect described in this study.

In albino mice, retinogeniculate axons incompletely segregate into eye-specific territories, even in normal activity conditions. Furthermore, epibatidine treatment induces fragmentation of the ipsilateral projection, a phenotype that is not observed in wildtype or EphB1 $1^{-1-}$ mice. The albino retina may exhibit altered physiological responses due to defects in proliferation and specification. This hypothesis is supported by an increased number of amacrine cells (which initiate the retinal waves during the first postnatal week) in albino rats (Mitrofanis and Stone, 1988). Future studies on neuronal activity in the albino retina should shed light on this intriguing phenotype.

The defects in RGC neurogenesis, molecular specification, axon guidance and targeting in the albino mouse could serve as reference points for rescue experiments and give insight into how melanin and the retinal pigment epithelium affect normal retinal development.

\section{References}

Apkarian P (1996) Chiasmal crossing defects in disorders of binocular vision. Eye 10:222-232.

Cang J, Rentería RC, Kaneko M, Liu X, Copenhagen DR, Stryker MP (2005) Development of precise maps in visual cortex requires patterned spontaneous activity in the retina. Neuron 48:797-809.

Creel DJ, Summers CG, King RA (1990) Visual anomalies associated with albinism. Ophthalmic Paediatr Genet 11:193-200.

Cronin CA, Ryan AB, Talley EM, Scrable H (2003) Tyrosinase expression during neuroblast divisions affects later pathfinding by retinal ganglion cells. J Neurosci 23:11692-11697.

Dräger UC (1985) Birth dates of retinal ganglion cells giving rise to the crossed and uncrossed optic projections in the mouse. Proc R Soc Lond B Biol Sci 224:57-77.

Dräger UC, Olsen JF (1980) Origins of crossed and uncrossed retinal projections in pigmented and albino mice. J Comp Neurol 191:383-412.

Feller MB, Wellis DP, Stellwagen D, Werblin FS, Shatz CJ (1996) Requirement for cholinergic synaptic transmission in the propagation of spontaneous retinal waves. Science 272:1182-1187.

García-Frigola C, Carreres MI, Vegar C, Mason C, Herrera E (2008) Zic2 promotes axonal divergence at the optic chiasm midline by EphB1dependent and -independent mechanisms. Development 135:1833-1841.

Guillery RW, Scott GL, Cattanach BM, Deol MS (1973) Genetic mechanisms determining the central visual pathways of mice. Science 179:1014-1016.

Hanson MG, Landmesser LT (2004) Normal patterns of spontaneous activity are required for correct motor axon guidance and the expression of specific guidance molecules. Neuron 43:687-701.

Herrera E, Brown L, Aruga J, Rachel RA, Dolen G, Mikoshiba K, Brown S, Mason CA (2003) Zic2 patterns binocular vision by specifying the uncrossed retinal projection. Cell 114:545-557.
Huberman AD, Feller MB, Chapman B (2008) Mechanisms underlying development of visual maps and receptive fields. Annu Rev Neurosci 31:479-509.

Ilia M, Jeffery G (1996) Delayed neurogenesis in the albino retina: evidence of a role for melanin in regulating the pace of cell generation. Brain Res Dev Brain Res 95:176-183.

Itoh K, Stevens B, Schachner M, Fields RD (1995) Regulated expression of the neural cell adhesion molecule L1 by specific patterns of neural impulses. Science 270:1369-1372.

LaVail JH, Nixon RA, Sidman RL (1978) Genetic control of retinal ganglion cell projections. J Comp Neurol 182:399-421.

Lee R, Petros TJ, Mason CA (2008) Zic2 regulates retinal ganglion cell axon avoidance of ephrinB2 through inducing expression of the guidance receptor EphB1. J Neurosci 28:5910-5919.

Le Fur N, Kelsall SR, Mintz B (1996) Base substitution at different alternative splice donor sites of the tyrosinase gene in murine albinism. Genomics 37:245-248.

Marcus RC, Wang LC, Mason CA (1996) Retinal axon divergence in the optic chiasm: midline cells are unaffected by the albino mutation. Development 122:859-868.

Mitrofanis J, Stone J (1988) Distribution of cholinergic amacrine cells in the retinas of normally pigmented and hypopigmented strains of rat and cat. Vis Neurosci 1:367-376.

Oetting WS (1999) Albinism. Curr Opin Pediatr 11:565-571.

Pak W, Hindges R, Lim YS, Pfaff SL, O'Leary DD (2004) Magnitude of binocular vision controlled by islet- 2 repression of a genetic program that specifies laterality of retinal axon pathfinding. Cell 119:567-578.

Petros TJ, Rebsam A, Mason CA (2008) Retinal axon growth at the optic chiasm: to cross or not to cross. Annu Rev Neurosci 31:295-315.

Pfeiffenberger C, Yamada J, Feldheim DA (2006) Ephrin-As and patterned retinal activity act together in the development of topographic maps in the primary visual system. J Neurosci 26:12873-12884.

Rachel RA, Dolen G, Hayes NL, Lu A, Erskine L, Nowakowski RS, Mason CA (2002) Spatiotemporal features of early neuronogenesis differ in wildtype and albino mouse retina. J Neurosci 22:4249-4263.

Rebsam A, Petros TJ, Mason CA (2009) Switching retinogeniculate axon laterality leads to normal targeting but abnormal eye-specific segregation that is activity dependent. J Neurosci 29:14855-14863.

Rice DS, Williams RW, Goldowitz D (1995) Genetic control of retinal projections in inbred strains of albino mice. J Comp Neurol 354:459-469.

Schaeren-Wiemers N, Gerfin-Moser A (1993) A single protocol to detect transcripts of various types and expression levels in neural tissue and cultured cells: in situ hybridization using digoxigenin-labelled cRNA probes. Histochemistry 100:431-440.

Sun C, Speer CM, Wang GY, Chapman B, Chalupa LM (2008) Epibatidine application in vitro blocks retinal waves without silencing all retinal ganglion cell action potentials in developing retina of the mouse and ferret. J Neurophysiol 100:3253-3263.

Williams SE, Mann F, Erskine L, Sakurai T, Wei S, Rossi DJ, Gale NW, Holt CE, Mason CA, Henkemeyer M (2003) Ephrin-B2 and EphB1 mediate retinal axon divergence at the optic chiasm. Neuron 39:919-935.

Williams SE, Grumet M, Colman DR, Henkemeyer M, Mason CA, Sakurai T (2006) A role for Nr-CAM in the patterning of binocular visual pathways. Neuron 50:535-547.

Xu HP, Furman M, Mineur YS, Chen H, King SL, Zenisek D, Zhou ZJ, Butts DA, Tian N, Picciotto MR, Crair MC (2011) An instructive role for patterned spontaneous retinal activity in mouse visual map development. Neuron 70:1115-1127. 